\title{
Algorithm of Intestinal Parasites Found at the Abass NDAO Hospital in Dakar
}

\author{
Pathé Thiongane $^{1}$, Amadou Ndiaye ${ }^{1 *}$, Arfang Diamanka ${ }^{3}$, Cheikhna Diebakhate $^{3}$, \\ France-Lyse Clotilde $\mathrm{Ba}^{3}$, Moustapha Diagne ${ }^{3}$, Ngor Faye ${ }^{3}$, \\ Pape Mbacké Sembene ${ }^{3}$ and Niama Diop Sall ${ }^{1,2}$
}

\section{${ }^{1}$ Abass NDAO Hospital, Department of Laboratories, Avenue Cheikh Anta Diop framed} between road Fann, the Road 50 and 67 of the Medina

${ }^{2}$ Faculty of Medicine, Pharmacy and Dentistry (FMPOS) in Dakar, Laboratory of Medical Biochemistry, UCAD

${ }^{3}$ Faculty of Science and Technology, Department of Animal Biology, Dakar-Senegal

*Corresponding author

\section{Keywords}

Abass NDAO Hospital, MCBA, prevalence, parasites, Stool and Ritchie's method

Article Info

Accepted: 04 May 2019 Available Online: 10 June 2019

\section{A B S T R A C T}

In Senegal, an efficient and effective struggle against intestinal parasites and there, their affections (bordering $40 \%$ of the global nature of the tropical diseases other than the malaria especially in the African regions), requires essentially a good knowledge on one hand of their frequencies and geographical distributions, and on the other hand of their assessments and epidemiological distributions, and on the other hand of their assessments and epidemiological evolutions. It is from this perspective; a study was led to Medical Center of Biology Analysis (MCBA) at Abass NDAO Hospital in Dakar during the first nine months of 2013. During this period every sample of stools underwent a macroscopic exam followed of the microscopic exam (each stool sample underwent a macroscopic examination followed by a direct microscopic examination and after concentration according to Ritchie's method). At the end of study, the exploitation of the data emanating from 125 patients got the following results: An overall prevalence of 16, 8\%. Variation of the parasitic carriage according to different parameters (month, age, sex, consistency of the stool and reason for diagnosis). Specific parasitic prevalences with 10,4\% for Entamoeba coli;2,4\% for Trichomonas intestinalis or Ascaris lumbricoides and 0,8\% for Entamoeba histolytica, Trichuris trichiura ,Strongyloides stercoralis ,Hymenolepis nana or Taenia sp;. Prevalence of the poly parasitism is $1,6 \%$ and an ascendancy of protozooses with a prevalence of $13,6 \%$ against $5,6 \%$ for helminthiasis.

\section{Introduction}

Due to their medical conditions and cosmopolitanism, which in certain parts of the world are favored by circumstances such as lack of hygiene and sanitation, lack of adequate sanitary facilities and climatic conditions; the parasitological parasites, thanks to the parasitological examination of human stools, constitute a veritable sanitary haven for people. Because of their presence in the human digestive tract, their proliferation 
and their future location, many of these parasites are subject to either simple asymptomatic portages or portages subject to considerable clinical manifestations. These intestinal parasites potentially belong to two systematic groups, Helminths and Protozoa (Rousset JJ, 1993).

The WHO estimates that more than 2 billion people in the world are affected by these parasites, 300 million people, seriously ill people, and 155,000 deaths a year due to these parasitic infections.. Especially in the tropics, these diseases are medically important because they account for nearly $40 \%$ of tropical diseases other than malaria. Remarkably, these parasitic diseases are found for the most part in African regions (WHO, 2012)

Senegal, a country with a tropical climate that, in addition to the habits and lifestyles of its people, combines all the physical and geographical elements (temperature, climate, rainfall, vegetation, sunshine and altitude) conducive to the maintenance and proliferation of parasites intestinal, is not spared the harmful impact of their affections and is a great home for them.

Also, an efficient and effective fight against these parasites and thus their affections requires primarily a good knowledge on the one hand of their frequencies and geographical distributions and on the other hand of their distributions and epidemiological evolutions. Having this information requires the relevance and permanence of the surveillance system through descriptive investigations. It is in this context that we conducted a study over a period of nine months (January to September 2013) at the Center for Analysis in Medical Biology (CABM) of the Abass Ndao Hospital Center (CHAN); in order to determine the overall prevalence of parasites, to estimate the frequency of parasite species and associations, and to evaluate parasite prevalence according to different parameters.

\section{Materials and Methods}

\section{Material}

We used various types of equipment, materials and consumables:

During sampling: transparent plastic pots that can be closed hermetically; plaster and marker for the labeling of sampling vessels;

During direct examination (fresh state and color): disposable gloves; agitator stick; physiological water and needle syringe; lugol $1 \%$ (dropper system); slides and lamellae of histology; black pencil and photonic microscope.

In enrichment or concentration technique (Ritchie method): glass rod to collect a portion of stool; footed glass helping to crush hard stools; plastic pots; gauze band ensuring sieving (obtaining the filtrate); $10 \%$ formalin solution as an aqueous dilution solution; graduated tubes with a conical bottom collecting the filtrate to be centrifuged; Ether; centrifuge; Pasteur pipettes; disposable gloves; slides and lamellae of histology; black pencil and photonic microscope. Freshly made saddles

\section{Study methods}

Each sample underwent macroscopic examination followed by microscopic examination.

\section{Macroscopic examination}

Visually, gross examination consists of detecting the consistency of the stool; the presence of mucus, mucus and / or blood and 
the possible presence of adult worms or cestode rings.

\section{Microscopic examination}

Microscopic examination involves a direct examination and a post-concentration examination according Ritchie method

\section{Direct examination in the fresh state and after staining}

This first part of the microscopic examination is as follows: a drop of saline is deposited in the center of the upper half of the slide and a drop of the lugol solution in the center of the lower half (the portion of the slide serving number the microscopic preparation being considered the lower end). By means of an applicator stick, a small portion of saddle is taken and mixed well with the drop of saline solution. In the same way, a small portion of sample is taken and mixed with the drop of the iodine-iodide solution ( $1 \%$ lugol). The mixtures obtained are covered with respective lamellae. The whole of the preparation thus carried out is observed under a light microscope first at the X10 objective for the identification and then at the X40 objective for the identification.

\section{Post-Concentration review: modified ritchie method}

This physic-chemical enrichment technique is concretized by proceeding as follows: about 2 to $5 \mathrm{~g}$ of stool are taken from several points of the sample in order to gradually dilute them using a glass stirrer in a solution of $10 \%$ formalin (in a proportion of $1 \mathrm{~g}$ of stool per $10 \mathrm{ml}$ of formolated water). The preparation is allowed to stand for a maximum of one minute (decanting large particles) before sieving through a suitably folded gauze band. $9 \mathrm{ml}$ of the liquid obtained is transferred into a graduated conical bottom tube in order to add $3 \mathrm{ml}$ of ether. The mixture is vigorously agitated to become homogeneous before centrifuging at $1500 \mathrm{rpm}$ for three (3) minutes. The tube will comprise, after centrifugation, four (4) layers whose three upper layers are to be eliminated by sudden reversal of the conical tube. The pellet is recovered using a Pasteur pipette and examined directly under the optical microscope between blade and coverslip to the objective $\mathrm{X} 10$ and then to the objective $\mathrm{X} 40$. If necessary, a drop of saline is added to the pellet.

\section{Results and Discussion}

Following these two categories of examinations (macroscopic and microscopic), different information was obtained. These data were entered on a computer and processed using the Excel spreadsheet. The khi2 test under MegaStat, allowed us to compare the results relative to the different variables, the p-value lower than 0.05 being considered statistically significant.

\section{Presentation of the results}

Of the 125 patients, 21 are carriers of one or more parasites, an overall parasite carrying prevalence of $16.8 \%$. Seven (7) male subjects are parasitized with a male prevalence of $13.21 \%$, and fourteen (14) female subjects is a female prevalence of $19.44 \%$. The chi-square test reveals that both characters (parasitism and sex) are statistically independent. p-value $=0.3567>0.05 ; \mathrm{ddl}=1$.

The monthly parasitic prevalences are reported in Figure 1, that of July (36.84\%) being the most consistent.

\section{Presentation of the results}

Of the 125 patients, 21 are carriers of one or more parasites, an overall parasite carrying 
prevalence of $16.8 \%$. Seven (7) male subjects are parasitized with a male prevalence of $13.21 \%$, and fourteen (14) female subjects is a female prevalence of $19.44 \%$. The chi-square test reveals that both characters (parasitism and sex) are statistically independent. p-value $=0.3567>0.05 ; \mathrm{ddl}=1$.

The monthly parasitic prevalences are reported in Figure 1, that of July $(36.84 \%)$ being the most consistent.

Eight species were identified, including
Entamoeba coli (vegetative and / or cystic) found in 13 individuals, a prevalence of $10.4 \%$ (Figure 2). In the case of systematic groups, helminths were recorded in 7 individuals, with a prevalence of helminthiases of $5.60 \%$ and protozoa in 17 individuals, ie a prevalence of protozooses of $13.60 \%$. However, two types of associations parasites were found: a case of biparasitism (prevalence equal to $0.8 \%$ ) with $S$. stercoralis and $H$. nana; and a triparasitism case (prevalence equal to $0.8 \%$ ) with $A$. lumbricoides, T. trichiura and E. coli.

Fig.1 Monthly parasitic prevalences

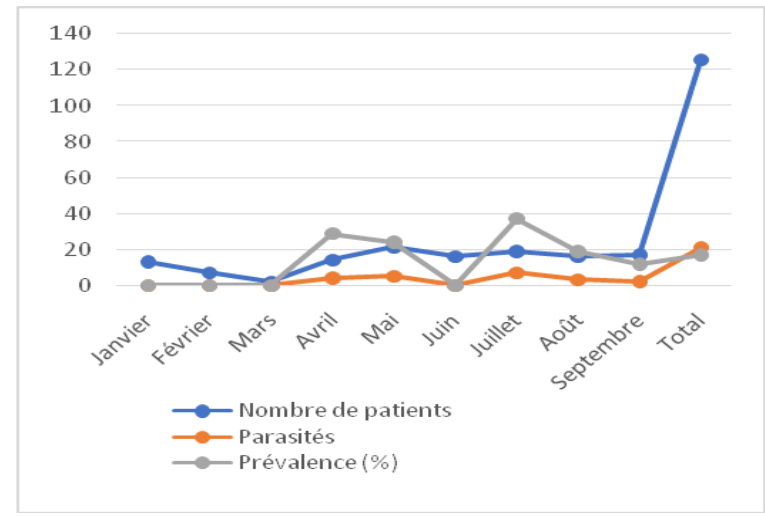

Fig.2 Prevalence of species encountered

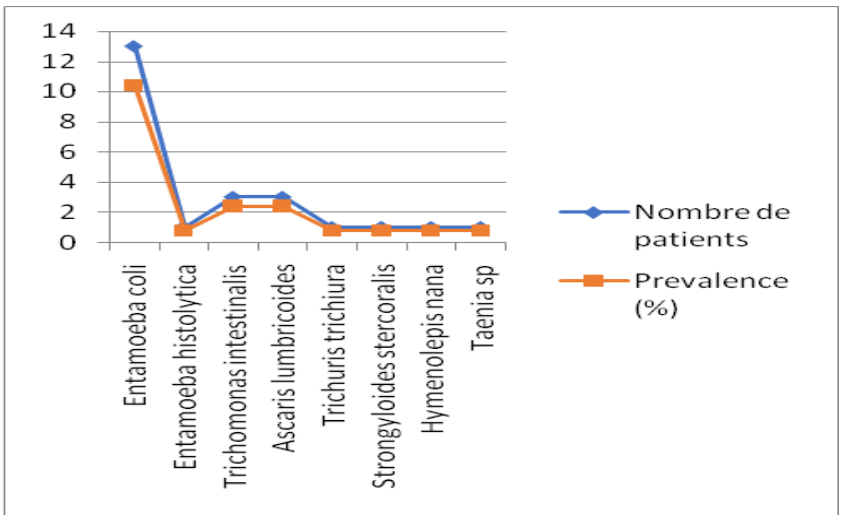


Fig.3 Prevalence of age cohorts

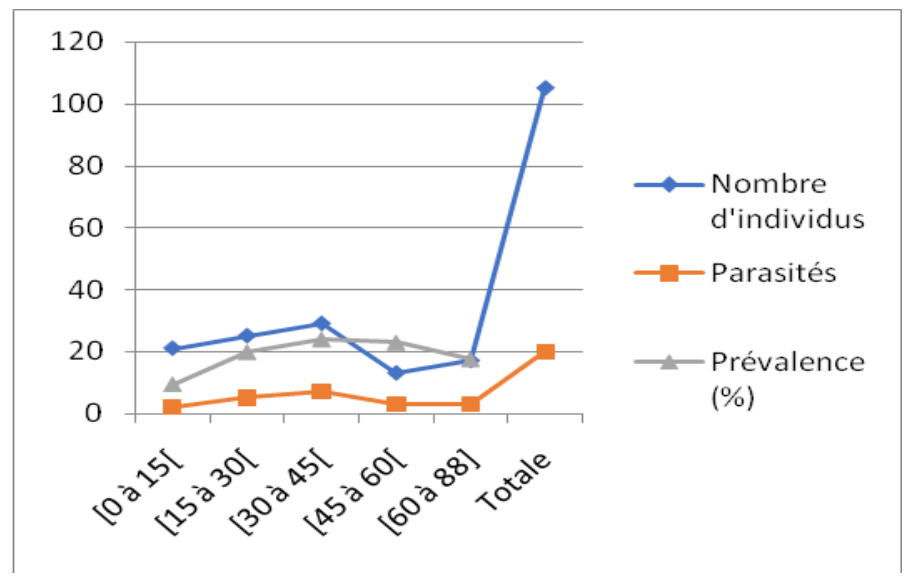

Fig.4 Prevalence according to the reason of prior diagnosis

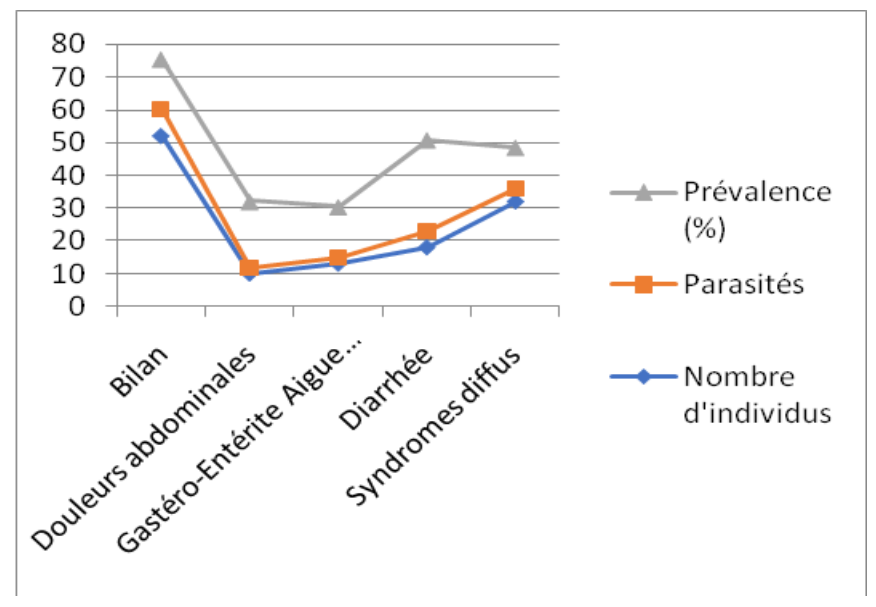

The infestation rate is higher in the age group [30 to 45 years [24.14\% (Figure 3). Age and parasitic carriage are, however, statistically independent ( $\mathrm{p}$-value $=0.7549$ and dof $=4)$. It appears that the parasite prevalence is $15.38 \%$ in individuals seeking a parasitological assessment and also $15.38 \%$ in subjects with GEA (Figure 4). Similarly, the diagnostic pattern and the parasitic carriage are statistically independent $(\mathrm{p}$-value $=0.7097$ and ddl $=4$ ).

The infestation rate estimated at $16.8 \%$ has just reached rates already recorded in the Dakar region (Ndiaye A., 2006 and Seck M.
C., 2012). Similarly, investigations concerning other areas of Senegal (Diongue O., 2001 and Gueye C., 2006) or other areas (Carvalo-Costa FA et al., 2007 and Nanfah WMP, 2008) have rates higher than the one we just found. On the other hand, it is higher than recently reported rates (Amine et al., 2008 and Ndayo, 2011).

Our prevalence, like the others, is essentially a matter of hygiene versatility, the lack of health information and education, and the quality and way of life of the population. Their difference is explained on the one hand by the nature of the copro-parasitological 
research techniques and on the other hand by the characteristics of the sample. Indeed, the infestation rate we found is slightly higher than that found at Khelcom (Thiam M., 1999), using only the direct examination.

Intestinal protozooses predominate in our study with a prevalence of $13.60 \%$ against $5.60 \%$ for helminthiases. Indeed, the detection of most protozoa (undoubtedly associated with transmission by drinks, hands or contaminated food) by standard techniques, contributes to this finding. Also, a suspicion of intestinal parasitosis generally elicits the use of antihelminthics and health organizations or actors work repeatedly against helminthiases. In Senegal, under the direction of the Ministry of Health, a deworming campaign is aimed at children aged 0 to 5 years, and soil-transmitted helminthiasis (considered as neglected tropical diseases) are at the origin of free chemo prevention.

Of the protozoa encountered in our study, E. coli has the highest prevalence rate of $10.4 \%$. This emanates from the fact that this nonpathogenic parasite, contaminated by hygienic failures, is naturally identified through its vegetative and / or cystic form. Helminthic species, A. lumbricoides predominates with a prevalence of $2.4 \%$. The other species found Taenia sp, H. nana, S. stercoralis and $T$. trichiura each have a prevalence of $0.8 \%$. The weakness of these prevalences relative to these different helminths reflects particularly the support that can emanate from certain specific research techniques (especially Kato and Bailenger) and the positive impact that some actions (sensitization and deworming campaign) could have on these parasites. Characteristically, our sample comprises in a large majority of individuals suggesting a parasitological balance (41.6\%).

There was a female infestation rate of $19.44 \%$ against $13.21 \%$ for the male one. Indeed, by their activities (garrotes, housewives, etc.), female subjects could be the most exposed to contamination. Also, individuals aged 15 to 60 years are the most parasitized with $20 \%$ for [15 to 30 years [, $24.14 \%$ for [30 to 45 years [and $23.08 \%$ for [ 45 to 60 years [. These people (pupils, garbage collectors, shopkeepers, garrote customers, etc.) could also be the most exposed to insalubrity, especially in urban areas.

Parasite prevalence appears to be higher in patients with diarrhea $(27.78 \%)$ and those with abdominal pain (20\%). This is because diarrhea and abdominal pain characterize intestinal disorders caused by intestinal parasites and are not medically taken lightly.

Also, the parasite prevalence followed a monthly fluctuation being more important in the months of July (36.84\%), April (28.57\%) and May (23.81\%), during the wet season, are more suitable for the maintenance and transmission of intestinal parasites (hence remarkable prevalences noted in August $(18.75 \%)$ and September (11.76\%)). Senegal is indeed a country with a tropical climate characterized by a short wet season (July to October).

It is concluded at the end of our study, that the rate of infestation by intestinal parasites remains significant in the Senegalese capital. It would be interesting to compare the type of parasitic disease according to the source, the use or not of antiparasitic agents for preventive purposes and dietary habits. This would correlatively allow the data obtained, to initiate better therapeutic pest coverage.

\section{References}

Amine M., Zougaghi L., El houdzi J. and Adarmouch L., 2008. Epidemiology of intestinal parasitic infections in children. Medical Expectation, Vol.15, No. 152, 477-482. 
Carvalo-Costa F. A., Gonçalves A.Q. and Lassance S.L., 2007. Giardia lamblia and other intestinal parasitic infections and their relationships with nutritional status in Brazilian Amazon. Revista do Instituto de Medicina Tropical Sao Paulo. Vol.49, No. 3, 147-153.

Diongue O., 2001. Qualitative and quantitative evaluation of parasitological examinations carried out in the laboratory of the Fatick health center from 1996 to 2000. Thesis Pharmacy. Dakar, No. 78, 73p.

Gueye C., 2006. Study of intestinal parasitosis detected at Amadou Sakhir Mbaye Hospital of Louga during the period 2004-2005.Thèse Pharmacie.Dakar, N $\circ 47,54-57$.

Nanfah W.M.P., 2008. Study of intestinal polyparasitism at INRSP in the district of Bamako-Mali. Thesis Pharmacy. Bamako, 106p.

Ndayo S.V.G., 2011. Intestinal parasitic carriage in children in Africa. Thesis Pharmacy. Lille: University of Lille 2, $106 \mathrm{p}$.
Ndiaye A., 2006. Contribution to the study of intestinal parasitosis at the Institute of Social Pediatrics of PikineGuédiawaye. Thesis Pharmacy. Dakar, No. 3, 60-63.

Rousset J-J., 1993. Practical Coproparasitology: Interest and Methodology - Notions on digestive tract parasites. Paris: ESTEM, 89 pages, ISBN 2-909455-15-7.

Seck M. C., 2012. Study of the prevalence of intestinal parasitosis in the commune of Dalifort (Department of Dakar, Senegal). Thesis, Pharmacy. Dakar, N $\circ$ 93, 75 pages

Thiam M., 1999. Prevalence of endemic parasitic diseases in the Khelcom area (Gossas department, Fatick region) Results obtained in Touba-khelcom (Quranic School No. 9). Thesis Pharmacy. Dakar, No. 74, 49-63.

WHO, 2012. Helminthiasis Control in School-Age Children: A Guide for Program Managers. 2eédition. Geneva: WHO Publishing, 75p.

\section{How to cite this article:}

Pathé Thiongane, Amadou Ndiaye, Arfang Diamanka, Cheikhna Diebakhate, France-Lyse Clotilde Ba, Moustapha Diagne, Ngor Faye, Pape Mbacké Sembene and Niama Diop Sall. 2019. Algorithm of Intestinal Parasites Found at the Abass NDAO Hospital in Dakar. Int.J.Curr.Microbiol.App.Sci. 8(06): 428-434. doi: https://doi.org/10.20546/ijcmas.2019.806.048 